\title{
UNILATERAL CYSTIC RENAL DISEASE WITH DIFFUSE INVOLVEMENT OF KIDNEY: A CASE REPORT
}

Manoj Hazarika ${ }^{1}$, Nabanita Deka², Gautam Goswami ${ }^{3}$

\section{HOW TO CITE THIS ARTICLE:}

Manoj Hazarika, Nabanita Deka, Gautam Goswami. “Unilateral Cystic Renal Disease with Diffuse Involvement of Kidney: A Case Report". Journal of Evolution of Medical and Dental Sciences 2014; Vol. 3, Issue 52, October 13; Page: $12217-12220$, DOI: $10.14260 /$ jemds/2014/3613

ABSTRACT: Unilateral renal cystic disease (URCD) is a rare, non-familial, non-progressive, unilateral cystic disorder of the kidney that is not associated with cysts or disorders in other organs and it is not related to other genetic cystic diseases. Only a few cases have been reported in the literature. We report a case of URCD in an adult male 49 years of age. Absence of a family history, benign clinical course and limitation of the disease to one kidney distinguish this condition from autosomal dominant polycystic kidney disease (ADPKD).

KEYWORDS: Unilateral renal cystic disease, autosomal dominant polycystic kidney disease.

ABBREVIATIONS: ADPKD (Autosomal dominant polycystic kidney disease) URCD (Unilateral renal cystic disease).

CASE REPORT: A 49 year-old man presented to surgery OPD with chronic vague pain in the right flank. There were no gross hematuria, fever and lower urinary tract symptoms. Other cardiovascular, respiratory, gastrointestinal, and neurologic symptoms were absent. No family illnesses including renal diseases were reported. Upon physical examination, blood pressure was 116/80 mmHg. Right kidney was palpable. Serum creatinine level and other blood tests showed normal findings. Patient had an ultrasound report done outside showing cystic lesions in right kidney. He was advised a CT abdomen for further management. The MDCT revealed an enlarged right kidney filled with variable sized well-marginated multiple uncapsulated cysts.

The cysts were found involving the kidney diffusely with small areas of residual normal renal parenchyma in the lower pole which were normally enhanced with contrast media. No enhancing soft tissue component or bands were seen within the cysts. Normal excretion of contrast was seen into the collecting system. No cysts were detected in left kidney or other intra- abdominal organs such as liver, pancreas or spleen. A diagnosis of unilateral cystic renal disease with diffuse involvement of right kidney was made with these imaging features.

DISCUSSION: Unilateral renal cystic disease (URCD) is rare, benign condition and first described in 1964 as unilateral polycystic renal disease. ${ }^{1}$ It is a multicystic disease, characterized by the replacement of one kidney by multiple cysts of varying sizes without the association of cysts in the contralateral kidney. The disease is non-familial, non- progressive and not related to autosomal dominant polycystic kidney disease (ADPKD). ${ }^{2}$

Levine et $\mathrm{al}^{3}$ proposed the term 'unilateral renal cystic disease ' as a distinct disease entity in 1989. Kohno and Yunoki² reported two patterns of unilateral cystic renal disease involvement as noted on imaging studies. In the diffuse form, as in our patient, multiple cysts are scattered throughout the affected kidney and in the segmental form, the cysts predominate in one region of the involved kidney. ${ }^{4}$ 
The morphologic feature of URCD is indistinguishable from that of ADPKD both radiologically and pathologically. However URCD has five aspects different from ADPKD: (1) unilateral localization, (2) negative family history, (3) no progression to chronic renal failure, (4) no cysts in other intraabdominal organs such as liver, spleen, or pancreas and (5) no disorders affecting other body organ systems. ${ }^{5}$

The clinical importance of URCD is to make a differential diagnosis of such abnormalities including multilocular cystic nephroma, cystic partially differentiated Wilms' tumor, segmental cystic dysplasia, and atypical presentation of polycystic kidney disease such as asymmetric evolution and mosaicism. ${ }^{6}$

The pathogenesis of this cystic renal disease is unknown. Since there is a morphological similarity of this cystic change to ADPKD, it is speculated that pathogenesis is similar. ${ }^{7}$ In URCD, cysts may be scattered diffusely throughout the kidney or involve a more localized portion of the kidney.

Therefore, the radiographic findings depend on the number and size of the cysts and the amount of normal renal parenchyma between the cysts. In either form, no distinct encapsulated renal mass is formed. It is this finding that is crucial in distinguishing it from a focal cystic mass such as multilocular cystic nephroma or cystic carcinoma. ${ }^{8}$

Multiple simple renal cysts and unilateral multicystic dysplastic kidney disease must be also considered in the differential diagnosis of the patients with URCD. In unilateral multicystic dysplastic kidney disease, the kidney is usually non- functioning, as the collecting system is usually atretic or obstructed. While in URCD, contrast excretion is seen in the collecting system which may be appearing normal or show displacement.

Sometimes, multiple simple cysts may be difficult to distinguish from URCD when the cysts are confined to one segment of the kidney, but they are less numerous than in URCD. Furthermore, unlike URCD where the cysts involve both the cortex and the medulla, they are predominantly located in the renal cortex. ${ }^{9}$ In conclusion; URCD is an entity that needs to be recognized by clinicians as a benign condition that does not require any specific treatment unless it causes clinical symptoms or hypertension. Only periodic imaging follow-up is necessary. ${ }^{5}$ Greater familiarity of this condition may help prevent its confusion with other cystic diseases of the kidney, each of which has a different management and prognosis.

Fig 1: Non contrast (A) and post contrast (B) axial MDCT reveals multiple cystic lesions involving diffusely the right kidney (thick white arrows).

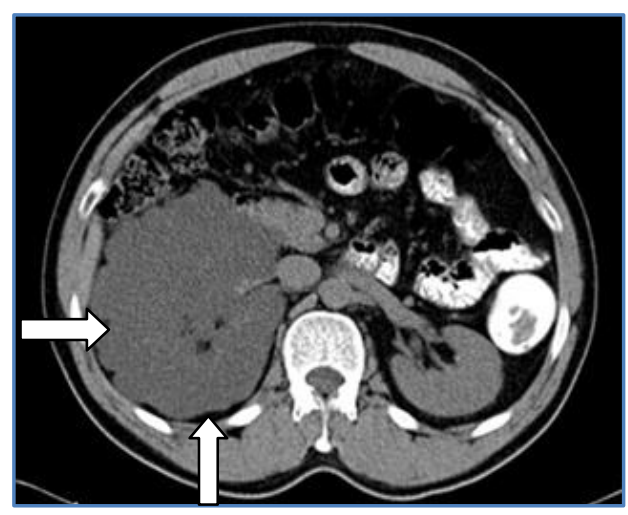

Fig. 1A

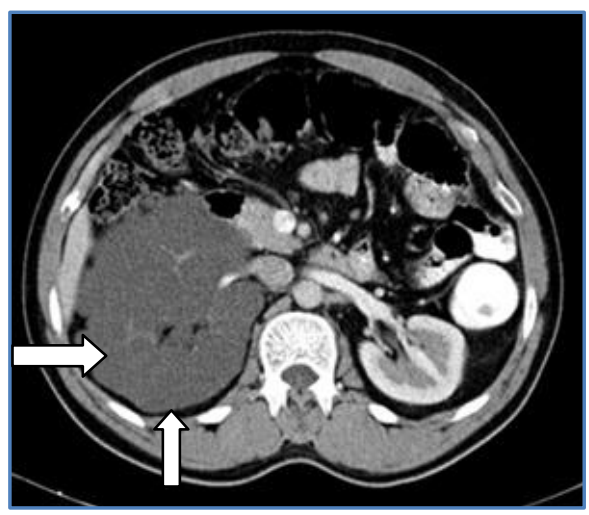

Fig. 1B 


\section{CASE REPORT}

Fig. 2: Post contrast MDCT axial image in (A) reveals normally enhancing renal parenchyma(shown in white arrow).Delayed axial MDCT image in (B) showing excretion of contrast in the calyces in both the kidneys (thin black arrows).

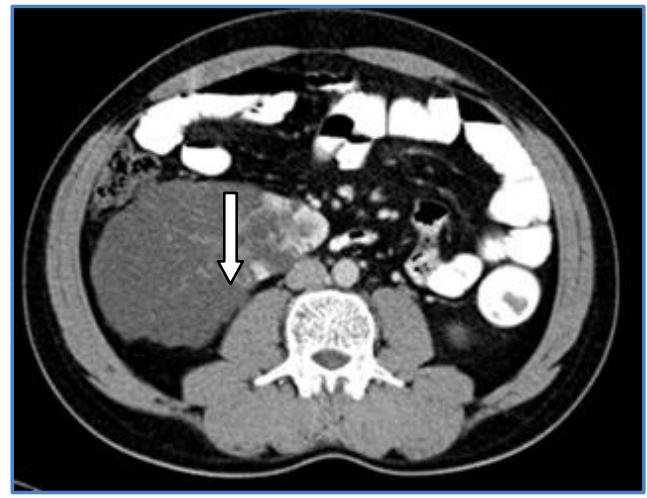

Fig. 2A

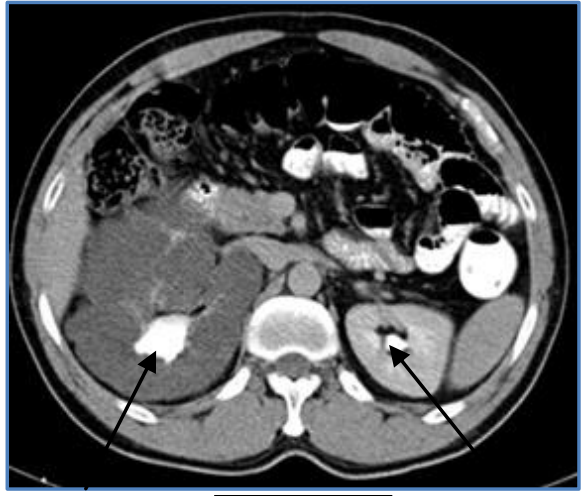

Fig. 2B

Fig. 3: Coronal MDCT images showing multiple cysts in the right kidney with diffuse involvement causing its enlargement (thick white arrows) No enhancing solid component seen within the cysts. The left kidney is normally seen (thin white arrow). Sagittal MDCT image(C) showing normally enhancing renal parenchyma in between the cysts (thin black arrow).

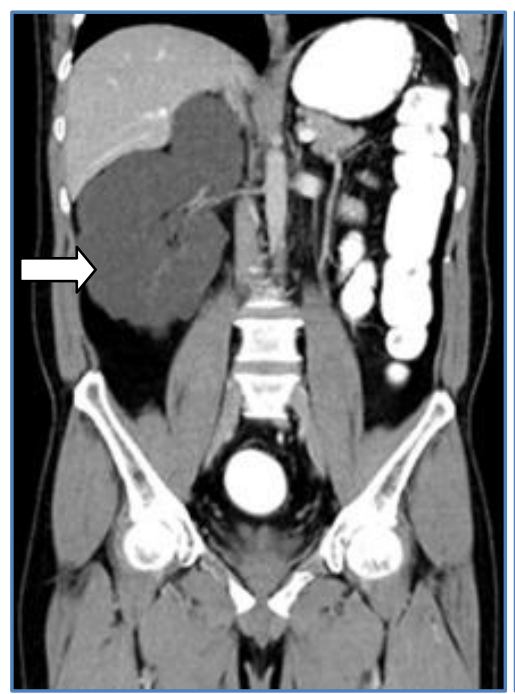

Fig. 3A

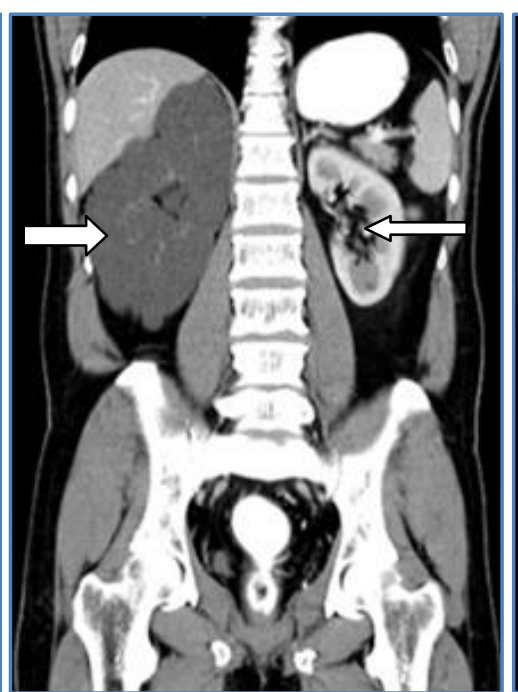

Fig. 3B

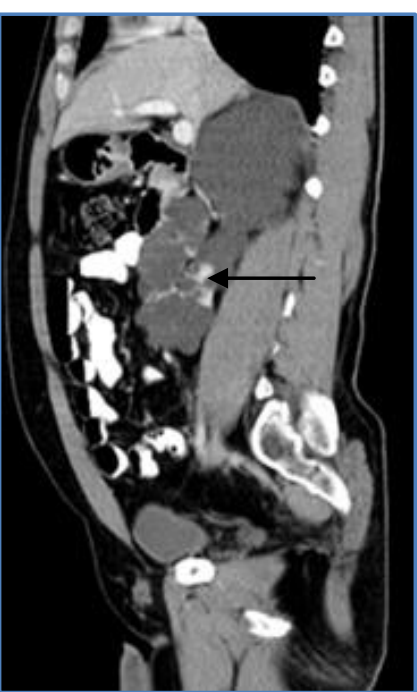

Fig. 3C

\section{REFERENCES:}

1. Park BS, Kim TH, Lim SJ, Lee HL, Jeon SH. Unilateral renal cystic disease. Korean J Urol 2007; 48: 652-6.

2. Kohno A, Yunoki M. Unilateral renal cystic disease. Radiat Med 1999; 17: 423-6.

3. Levine E, Huntrakoon M. Unilateral renal cystic disease: CT findings. J Comput Assisted Tomogr 1989; 13: 273-276. 
4. Ding Y, Chen L, Deng FM, Melamed J, Fan R, Bonsib S, et al. Localized cystic disease of the kidney: Distinction from cystic neoplasms and hereditary polycystic diseases. Am J Surg Pathol 2013; 37: 506-13.

5. Bum Soo Park, Tae-Hwan Kim, Sung-Jig Lim et al. Unilateral Renal Cystic Disease. Korean J Urol 2007; 48: 652-654.

6. Eun Hui Bae, Young-Hwan Hwang, Soo Wan Kim. Unilateral renal cystic disease in the right kidney. Int Braz J Urol. 2013; 39: 435-7.

7. Gouldesbrough DR, Fleming S. Unilateral and segmental localized polycystic kidney disease. J Clin Pathol. 1998; 51: 703-5.

8. Chrystia M Slywotzky, Morton A Bosniak. Localized Cystic Disease of the Kidney. AJR 2001; 176: 843-849.

9. Aynur Solak, Mehmet Serkan Gür, Berhan Genç et al. Localized Cystic Disease of the Kidney: A Rare Cause of Hypertension in a Young Adult. Journal of Clinical Imaging Science 2013; 3: 33.

\section{AUTHORS:}

1. Manoj Hazarika

2. Nabanita Deka

3. Gautam Goswami

\section{PARTICULARS OF CONTRIBUTORS:}

1. Assistant Professor, Department of Radiology, Gauhati Medical College \& Hospital, Guwahati, Assam, India.

2. Assistant Professor, Department of Radiology, Gauhati Medical College \& Hospital, Guwahati, Assam, India.

3. Professor and HOD, Department of Radiology, Gauhati Medical College \& Hospital, Guwahati, Assam, India.

\section{NAME ADDRESS EMAIL ID OF THE CORRESPONDING AUTHOR:}

Dr. Manoj Hazarika, Assistant Professor, Department of Radiology, Gauhati Medical College \& Hospital, Guwathi-781032, Assam, India.

Email: manojhazarika23@gmail.com

Date of Submission: 22/09/2014. Date of Peer Review: 23/09/2014. Date of Acceptance: 07/10/2014. Date of Publishing: 13/10/2014. 